\title{
La segmentación laboral: hacia una tipología del ámbito productivo
}

\author{
Pedro López \\ Faustino M iguélez \\ Andreu Lope \\ Universitat Autònoma de Barcelona. D epartament de Sociologia \\ 08193 Bellaterra (Barcelona). Spain \\ Xavier Coller \\ Yale University. D epartment of Sociology
}

\section{Resumen}

Este artículo toma como temática central el mundo productivo y más concretamente la segmentación del mercado laboral, construyendo una tipología en esta dirección. D icha tipología es la base de referencia, dentro del ámbito productivo, para establecer y analizar las relaciones entre dicho ámbito y el reproductivo a partir de la información proveniente de una encuesta social, y por tanto, contando con datos cuantitativos y con sus métodos y técnicas correspondientes. El interés de esta tipología, además de su propia construcción, es triple: primero, porque en su elaboración no sólo intervienen indicadores y variables propias del mundo de la empresa, sino también otros datos contextuales; segundo, porque posibilita la ampliación de la tipología a otras poblaciones no ocupadas como parados, inactivos, amas de casa, y tercero, porque permite caracterizar con variables sociodemográficas y otras ligadas a los estilos de vida los tipos de segmentación laboral.

Este artículo es parte de una investigación cuyos resultados aparecen en este mismo número de PAPERS. El artículo tiene autonomía y entidad por sí mismo pero la lectura del resto de los artículos de este número facilitará su contextualización.

Palabras clave: mercado de trabajo, segmentación, trabajo productivo, metodología, tipología.

\section{Abstract}

The central topic of this article is the world of production, and more specifically the segmentation of the labour market, for which a typology is constructed. This typology is the basis of reference within the production environment for establishing and analysing the relations between this environment and the environment of reproduction, using the information from a social survey and therefore applying quantitative data with the corresponding methods and techniques. In addition to its value in itself, this typology is of additional interest for three reasons: first, because it involves not only indicators and variables characteristic of the business world but also other contextual data; second, because it allows the typology to be extended to non-employed persons, such as the unemployed, the inactive and housewives; and third, because it allows the types of labour segmentation to be categorised with socio-demographic variables and other variables related to lifestyles.

This paper is part of a research work whose results are in this PAPERS volume. The paper is autonomous but the reading of the other papers of this volum can help to contextualize it.

Key words. labour market, segmentation, environment production, methodology, typology. 


\section{Sumario}

0. Introducción

1. El modelo de segmentación
2. Análisis de la segmentación del mercado de trabajo

Bibliografía

\section{Introducción}

Presentamos el planteamiento, los procedimientos y los resultados más relevantes del proceso de construcción de una tipología del ámbito del trabajo productivo desde la perspectiva teórica de la segmentación del mercado de trabajo.

La exposición que realizamos consta de dos partes principales. En una primera se relatan los contenidos fundamentales que justifican una aproximación desde la perspectiva de la segmentación. En ella se definen los rasgos del modelo general sobre el que hemos razonado en el estudio del trabajo productivo: el concepto de segmentación laboral y su dimensionalización, así como las hipótesis que guían la descripción y explicación de las principales transformaciones del trabajo en su relación con el mercado de trabajo y la estructura social, y que se expresan a manera de síntesis o de hipótesis general en la plasmación de una tipología de segmentación laboral.

U na segunda parte preten de precisamente obtener evidencia empírica de una tipología de segmentación del mercado de trabajo. Para ello nuestras reflexiones no sólo han pretendido expresar los contenido sustantivos de esta perspectiva teórica, sino que han querido probar distintos métodos también con una finalidad de análisis metodológico en la construcción de tipologías y que resulta por tanto complementario al propio análisis de la segmentación.

\section{El modelo de segmentación}

En nuestro ámbito de estudio nos interesa abordar el análisis de las transformaciones - y permanencias - más relevantes en el trabajo. Entenderemos el trabajo como un fenómeno social sujeto a la influencia de las estrategias de los diversos actores sociales, desde la institución familiar hasta el Estado y, en particular hasta las estrategias de los trabajadores, los empresarios, y de sus representantes respectivos: sindicatos y organizaciones empresariales. Asimismo, dentro del trabajo nos referiremos concretamente al empleo, esto es, aquellas actividades remuneradas sujetas a los mecanismos de mercado. Excluimos, por tanto, todas aquellas tareas que se engloban dentro del llamado «trabajo doméstico o de la reproducción», así como las diversas formas de trabajo voluntario o social (Pahl, 1990 y 1991). Partimos, igualmente, del supuesto de que la actividad trabajo-empleo resulta fundamental en la configuración de algunos aspectos clave de las condiciones de vida.

Para el estudio de la segmentación del mercado de trabajo nos centraremos en este estudio en el contexto español y más especificamente en el catalán, 
desde finales de los años setenta. Se trata de un período que presenta un doble interés. Por un lado, nos sitúa en una fase de reestructuración productiva y organizativa y, por otro lado, es a partir de esos años que podemos empezar a hablar, en nuestro país, de intervención autónoma en el mercado de trabajo de las organizaciones empresariales y sindicales.

\subsection{La perspectiva teórica de la segmentación}

Para desarrollar la propuesta anterior emplearemos el marco conceptual y analítico que ofrece el modelo o la perspectiva teórica de la segmentación del mercado de trabajo. Esta perspectiva intenta explicar las permanencias y transformaciones en el trabajo a que da lugar la reestructuración productiva y organizativa a las que hacíamos referencia, considerando que las organizaciones que actúan en el terreno económico (entendidas en sentido amplio) crean y desarrollan puestos de trabajo diferenciados entre sí. N os referimos aquí básicamente a los empresarios, pero también a los sindicatos, cuya mutua interacción en la negociación colectiva o en la denominada «concertación social» coadyuva a configurar unas determinadas características de los puestos de trabajo (respecto a horarios y jornada laboral, salarios, condiciones de trabajo, etc.). En la misma línea situamos la intervención específica del Estado, ya sea como copartícipe de la concertación, como empleador, 0 a través de su producción normativa en materia laboral.

Este enfoque entiende que la estructura actual del mercado de trabajo permite diferenciar a la población en niveles o segmentos con condiciones de trabajo (y de vida) diferenciados según el lugar en que se ubiquen en el mismo. Se configuran, pues, segmentos diferenciados de puestos de trabajo, cada uno de ellos con características propias y homogéneas y, a su vez, distintas del resto de segmentos. La estructura e interrelación de tales segmentos es tal que a las personas les resulta difícil ubicarse en un segmento distinto al que sus características en relación con el trabajo le atribuyen de forma tendencial. Por su parte, los diversos segmentos se diferencian entre sí, jerárquicamente y en función del diseño diferencial de tales puestos que realizan las empresas, por cuestiones como: el nivel salarial, la seguridad en el empleo o las posibilidades de promoción y de establecimiento de carreras profesionales que posibilitan los puestos de trabajo que se inscriben en cada segmento. Sin embargo, cabe puntualizar que los segmentos no se construyen de una vez por todas. Por el contrario, los contenidos, los límites y la distancia entre segmentos varía según el contexto socioeconómico en que nos situemos. Se trata, por tanto, de procesos de segmentación y no de real idades prefijadas de antemano sujetas a modificaciones mecánicas.

\subsection{Construcción del modelo de segmentación}

La construcción del modelo que presentamos se basa en una serie de investigaciones, tanto teóricas (Piore, 1983; D oeringer y Piore, 1983 y 1985; G ordon, Edwards y Reich, 1986), como empíricas (Kern y Schumann, 1988; Recio, 1988 
y 1991; M iguélez, 1989; M iguélez y Torns, 1992; Villa, 1990; López, 1994 y 1996), tratándose en cierto modo de una herramienta de análisis probada en la descripción de la multiplicidad de situaciones con que las personas acceden al mercado de trabajo (cualificación profesional, sexo, etnia, edad, nivel educativo formal, procedencia socioeconómica, etc.) y de las posibilidades que de ahí derivan para insertarse en uno u otro segmento del mercado laboral.

Básicamente partiremos del modelo y la tipología propuestos por autores de la escuela radical americana (G ordon, Edwards y Reich, 1986) donde se plantea la existencia de tres segmentos, jerárquicamente ordenados en cuanto a la calidad de los empleos que se ubican en ellos y a la «capacidad de mercado", que proporcionan a sus ocupantes: el segmento primario independiente, el segmento primario dependiente o subordinado, y el segmento secundario ${ }^{1}$.

También cabe resaltar el análisis global de la actividad productiva que aquí se efectúa. $\mathrm{H}$ abitualmente, las formulaciones teóricas y sobre todo los estudios empíricos centran su interés en el sector industrial de la economía. En esta investigación, por el contrario, se considerará todo el conjunto de los puestos de trabajo, incluyendo situaciones de empleo sumergido.

Los ejes básicos para construir el modelo de segmentación son dos. El primero se refiere a las características de los puestos de trabajo en sí: incluyen los rasgos fundamentales que diferencian unos puestos de trabajo de otros. EI segundo tiene que ver con las características del contexto en que se desarrolla la configuración de dichos puestos. Veamos cuales son esos rasgos para cada uno de los dos casos.

\subsubsection{Características de los puestos de trabajo}

Para la configuración de cada uno de los segmentos se consideran cuatro tipos de características, dimensiones o elementos de los puestos de trabajo. Se establece como hipótesis que son esas características las que más nítidamente contribuyen a diferenciar unos puestos de trabajo de otros.

- Estabilidad versus inestabilidad en el empleo

En este caso nos interesa considerar la distinta capacidad para mantener el empleo que tienen los diversos colectivos de trabajadores. La máxima estabilidad se ejemplificaría en la figura de los técnicos de grado superior y en la de los funcionarios (por sus capacidades técnicas y por la seguridad ocupacional que otorga la oposición ganada); y la máxima inseguridad la encontraríamos en las personas en situación de paro de larga duración. El grado

1. En otro lugar (M iguélez, 1990) hemos considerado una novedad respecto a ese modelo, al margen de la adaptación de sus características intrínsecas a la realidad del mercado de trabajo catalán y español. Así, constatamos la presencia de un nuevo segmento, el marginal, junto a los ya mencionados. La inclusión de este segmento queda justificada por la necesidad de considerar la relación entre empleo y desempleo, ya que la misma tendencia a que determinados colectivos aparezcan adscritos a uno u otro segmento se observa respecto a la mayor o menor permanencia en situación de desempleo por parte de grupos concretos y a la oscilación entre empleo y paro que manifiestan otros colectivos. 
estable supone, en el mundo laboral actual, una posición social de seguridad y distinción.

D icha seguridad puede venir dada por tres tipos de factores. Uno de carácter legal, es decir, por la naturaleza de la relación contractual que regula la prestación laboral. El segundo factor se refiere a la solidez del propio puesto de trabajo. El tercer factor a considerar es la capacidad de negociación respecto a unas determinadas condiciones de realización efectiva del trabajo.

- Cualificación y promoción versus no cualificación y estancamiento profesional En este segundo aspecto de características diferenciadoras de puestos de trabajo nos referiremos a todo lo relacionado con los distintos niveles de cualificación profesional que requieren los puestos de trabajo; cualificaciones que suelen llevar aparejadas distintas posibilidades de promoción y expectativas profesionales.

Consideraremos los tipos de conocimientos técnicos necesarios para desarrollar una tarea, así como la existencia o no, y su grado, de la intervención personal (autonomía) en la planificación y ejecución de una actividad y el grado de control que pueda ejercerse sobre la misma². Entenderemos que la posibilidad de promoción está vinculada, entre otras cuestiones, a la cualificación «real» y a la «capacidad de mercado» o de negociación de que se dispone en relación con dicha cualificación.

- Ingresos

Aquí tendremos en cuenta la cuantía de las percepciones ${ }^{3}$. Los ingresos constituyen un aspecto importante de las condiciones del puesto de trabajo que se ocupa en la medida en que el trabajo asalariado implica una jerarquía de remuneraciones; se considera que estas diferencias reflejan una valoración diferenciada de la fuerza de trabajo y una situación desigual en el mercado de trabajo.

\subsubsection{Características contextuales}

Los rasgos fundamentales a que nos hemos referido para caracterizar los distintos puestos de trabajo acaban de perfilarse al situarlos en el contexto en que se desarollan y concretan. Para delimitar este contexto tendremos en cuenta los siguientes factores:

2. En este sentido se dirige el concepto de cualificación efectiva que desarrolla A. Lope (1996: 49-58).

3. O tros indicadores adicionalmente podrían dar razón de su evolución y de las garantías de su mantenimiento: las formas en que se percibe la remuneración, entendiendo que las más seguras proporcionan mayor estabilidad; el tipo de pago (por horas, semanas, meses... ), y la existencia o no de incrementos salariales pactados individual o colectivamente, es decir, el grado en que puede asegurarse el mantenimiento de la capacidad adquisitiva de los salarios. La existencia o no en la empresa de ventajas social es concretas («weffare empresarial»), o de determinadas formas de salario indirecto (primas, incentivos de diverso tipo... ), son otros elementos que ampliarían este apartado. 
- Existencia versus inexistencia de derechos laborales y sindicales

Esta característica diferenciadora entre puestos de trabajo se refiere a factores vinculados a la posibilidad de regulación y control sobre las condiciones de realización del trabajo (posibilidad de negociación colectiva, de contar con representantes del conjunto de los asalariados, etc.). La existencia de determinados derechos laborales y sindicales contribuye a configurar mayor seguridad en el empleo (y a dotarlo de mayor «calidad») 0, cuando menos, mayores garantías en caso de pérdida del mismo. Es, por otra parte, un elemento que contribuye a configurar la «solidez» de los puestos de trabajo antes citada, por cuanto los mecanismos colectivos de regulación y control sobre el trabajo posibilitan, frecuentemente, la formalización de medidas tendentes a fomentar la cualificación y la formación de los asalariados.

- El sector de actividad

Interesa definir si se trata de un sector consolidado o no, en expansión o en crisis. Pese a que existan puestos de trabajo con características comunes, las diferencias entre ellos pueden ser grandes de acuerdo con su posición en el conjunto del sistema productivo y de tratarse de sectores «punta» 0 en crecimiento 0 , por el contrario, de sectores que se encuentran en declive o en una posición subordinada en el contexto económico (Kern y Schumann, 1988).

- El tipo de empresa

Se distinguen las empresas según su titularidad, esto es, públicas o privadas. La titularidad nos permite ilustrar desde una aproximación global y contextual la existencia de diferencias en las estrategias empresariales que tie nen importantes consecuencias en el diseño de los puestos de trabajo, en particular, se entiende que la empresa pública ofrece una mayor seguridad y garantías de sus puestos de trabajo y del empleo de quienes los ocupan.

- El tamaño dela empresa

H abitualmente, la seguridad y estabilidad en el empleo se asocian al tamaño de la empresa en cuestión. Sin embargo, la relación no es lineal. Aparece mediatizada por el sector de actividad y el tipo de relaciones laborales existente en la empresa. Además, los procesos de diferenciación de los puestos de trabajo observados en la gran empresa, contribuyen a desdibujar el peso del tamaño de la empresa en la segmentación, al menos para determinados colectivos. Con todo, dado el tejido empresarial de nuestro país, con abundante presencia de medianas, pequeñas y pequeñísimas empresas, y el grado de cobertura de la negociación colectiva y de la presencia de representación laboral (escasos en la pequeña empresa), hacen que siga siendo importante el considerar esta variable. El análisis de las distintas políticas empresariales relativas a diferentes puestos de trabajo y colectivos de asalariados, ha proporcionado fecundas aportaciones a partir, por ejemplo y sobre todo para las grandes empresas, del concepto y estudio que desde el 
enfoque de la segmentación se realiza de los mercados internos de trabajo (0 sterman, 1988). Por otra parte, prácticas empresariales relativamente novedosas, como la «descentralización productiva», han originado relaciones de nuevo tipo entre la segmentación y el tamaño de la empresa.

La combinación de las características de los puestos de trabajo junto a las consideraciones contextuales, nos permite elaborar una tipología sobre la segmentación del mercado de trabajo ${ }^{4}$. La construcción de una tipología de segmentación ya ha sido experimentada con anterioridad a partir de los datos obtenidos en la Enquesta M etropolitana de Barcelona del año 1985 - repetida en 1990- (M iguélez, 1989; M iguélez y Torns, 1992). Tal tipología distinguía, a partir de un ejercicio de atribución de combinaciones de características definitorias, cuatro segmentos: primario independiente, primario dependiente, secundario y marginal. Ello no impide la posibilidad de perfeccionar dicha tipología a partir de la inclusión de otros elementos, ni la posibilidad que mostraremos de utilizar diferentes procedimientos para su construcción.

\subsection{Hipótesis relativas al modelo propuesto}

Las hipótesis que se proponen buscan la explicación de las características fundamentales que hoy presenta el empleo en un contexto social, económico y político definido que es el de nuestro país. Al mismo tiempo, tiene en cuenta las aportaciones teóricas y los resultados registrados en otros países. La investigación se guía por las siguientes hipótesis relativas al contenido y a los cambios que experimenta la estructura de la segmentación laboral:

- Una hipótesis general y central desde la perspectiva de la segmentación es la configuración de una tipología de segmentos o de situaciones de empleo que estructuran un mercado laboral definido por la división del empleo asalariado en dos segmentos, un segmento primario y otro secundario, el primero de los cuales comprende, a su vez, la distinción entre un segmento primario independiente y otro dependiente. El segmento primario se caracteriza por recoger las situaciones de empleo más estable características de los mercados internos de las grandes empresas y del sector público que se corresponden con puestos de trabajo centrales para la actividad de la empresa. La capacidad de negociación de los trabajadores de este segmento les

4. La tipología se refiere exclusivamente a los ocupados asalariados. En un momento del análisis tomaremos en consideración, también, a los no asalariados, si bien la heterogeneidad de las condiciones que presentan sus puestos de trabajo 0, mejor, sus actividades específicas, así como su condición de autoempleados, hace difícil incluirlos en el análisis específico que permite el modelo teórico de la segmentación. Su inclusión se justifica desde la perspectiva y los objetivos más globales de la investigación que persiguen vincular los ámbitos productivo, reproductivo y de vida cotidiana. 
dota de una garantía de mejores condiciones laborales y la regulación de las mismas, con claros mecanismos de promoción. El segmento secundario se definiría por la inestabilidad del empleo resultado de las estrategias de externalización que configura empleos con bajos niveles de cualificación y que no posibilitan su adquisición, con malas condiciones laborales, localizados en pequeñas empresas donde se carece de capacidad de negociación. En este segmento cabría incluir también el fenómeno de la economía sumergida. Un segunda división se establece en el interior del segmento primario resultado de estrategias de flexibilización y control de la fuerza de trabajo. Esta división tiende a diferenciar un segmento primario independiente, de empleo estable con los mayores niveles de cualificación, mejor remunerados y más enriquecedores en correspondencia con la centralidad de los puestos de tra bajo que se ocupan. El segmento primario dependiente contempla los puestos estables de menor cualificación que implican tareas más rutinarias y específicas, y el mantenimiento de unas buenas condiciones de trabajo que resulta de una capacidad de negociación de sus trabajadores.

- Adicionalmente cabe considerar una tendencia hacia el aumento de segmentos por un proceso de mayor diferenciación. El segmento primario dependiente tiende a debilitarse. Por el contrario, el secundario tiende a ampliarse al tiempo que se diversifica incluyendo a nuevos colectivos. C rece de forma lenta el segmento primario independiente con diferenciaciones en su seno que permiten plantearse la posibilidad de un doble segmento en el mismo. Al mismo tiempo que se acrecientan las distancias entre ellos en términos de las condiciones de realización efectiva del trabajo, de las cualificaciones requeridas, etc.

- La diferenciación del mercado de trabajo que nos apunta la perspectiva de la segmentación no es de ahora, pero sus formas actual es tienen que ver en relación con las estrategias empresariales: la segmentación de los puestos de trabajo se desarrolla bajo las exigencias de las empresas, especialmente de las grandes, en relación con el hecho de lograr una gestión laboral más controlada, productiva y, hoy en particular, más flexible. N o existe una relación determinista entre el tamaño de la empresa y la adscripción a uno u otro segmento, aunque sí una tendencia que relaciona a las empresas de menor tamaño con posibilidades de mayores dosis de inseguridad en el empleo, dada la tradicional menor capacidad de control y de garantías sobre el trabajo de que disponen los ocupados en ese tipo de empresas. Al mismo tiempo, y como novedad con respecto a períodos anteriores, la posibilidad de inseguridad aparece también en la gran empresa, como consecuencia de la aplicación de determinadas estrategias empresariales. El segmento secundario se extiende, por tanto, en el interior de las grandes empresas.

- También está en relación con las estrategias sindicales y los tipos de relaciones laboral es: las estrategias sindicales contribuyen a la diferenciación de los puestos de trabajo y por tanto a la segmentación. A nivel macro, a través de su intervención en la concertación, en acuerdos sociales 0 , más recientemente, 
en acuerdos específicos con el Estado o las organizaciones empresariales. A nivel de empresa, a través de la negociación colectiva y de la posibilidad de controlar sus resultados, así como de la existencia de garantías sindicales. LoS diversos modos en que se pueden concretar en las empresas las relaciones empresa/trabajadores (y en especial con los representantes de éstos), contribuyen a concretar tendencias específicas de la segmentación laboral.

- No podemos olvidar tampoco la incidencia de las políticas laborales de la Administración en las formas de segmentación. Podemos distinguir dos direcciones de esas políticas: a) La Administración como reguladora del mercado de trabajo: las nuevas formas de contratación favorecen de forma clara el aumento de la contratación no estable, lo que puede contribuir a incrementar la inseguridad en el empleo, y no sólo en la pequeña o mediana empresa sino también en la grande para determinados colectivos, con la consiguiente posibilidad de aparición de núcleos de empleo inseguro en ésta última. b) La Administración como empleadora: el presumible mantenimiento de políticas públicas para satisfacer las propias necesidades de la Administración en materia de empleo, puede favorecer el incremento del segmento primario independiente, por medio de la creación de puestos de trabajo cubiertos por técnicos y profesionales de los servicios públicos.

- La innovación tecnológica también se relaciona con la segmentación, aunque no repercute de forma mecánica ni unidireccional. Sus efectos actúan en una doble dirección. Por un lado, puede hacer que los puestos de trabajo requieran mayor cualificación profesional y posibilidades de intervención y control de los asalariados sobre el propio trabajo. Pero, por otro lado, puede actuar en sentido contrario, restando cualificaciones y capacidad de intervención y control. Asimismo, la innovación tecnológica, en ocasiones acompañada de innovaciones organizativas igualmente importantes, puede influir, también de forma variable, en la seguridad de los puestos de trabajo afectados por ella.

La perspectiva de análisis de la segmentación subraya también que hay dife rencias sociales entre los trabajadores, tanto en las condiciones en que realizan su trabajo como en sus condiciones de vida, en tanto que hay diferencias estructurales importantes en los trabajos a través de los cuales obtienen sus ingresos. Esta proposición nos permite plantear las implicaciones de la segmentación del trabajo sobre las desigualdades en el acceso al empleo y a los diversos tipos de puestos de trabajo que lo conforman. D ado que existen puestos de trabajo distintos, unos más consistentes y ricos que otros, unos donde existen mayores garantías y derechos que en otros, etc., las preguntas que se plantean son: ¿cómo se consigue el acceso a uno u otro puesto de trabajo? y ¿quiénes son las personas y los colectivos que acceden a cada uno de ellos? En la línea de atender a tales preguntas, avanzamos las siguientes hipótesis:

- Se produce un acceso desigual a los puestos de trabajo, pero no de forma indiscriminada. Existen personas o colectivos más proclives que otros a 
ocupar unos determinados segmentos. Según las características personales y sociales que tienen las personas, éstas pueden permanecer indefinida mente en un segmento o pueden ubicarse en él sólo de forma coyuntural. En este segundo caso, el cambio puede producirse tanto en forma de ascenso como de descenso.

- La permanencia en uno u otro segmento tiene incidencia sobre las condiciones de vida, las perspectivas de futuro y las actitudes de las personas y los colectivos que se insertan en ellos. Esto implica que las divisiones socia les son explicables, siquiera parcialmente, por la segmentación del mercado de trabajo.

- Podemos buscar los factores que con mayor intensidad pueden influir en el acceso a uno u otro tipo de puestos de trabajo en aquéllos de índole social y personal: sexo, edad, cualificación profesional, nivel educativo, etnia, etc. I gualmente, estos factores tienen un rol destacado en la exclusión, en forma de paro, de determinados grupos del mercado de trabajo. Los datos que proporciona la estructura del mercado de trabajo español y catalán respecto a las elevadas tasas de desempleo que se dan entre las mujeres y los jóvenes son indicativos de esta cuestión. Pero hemos de profundizar más para captar el grado en el que influyen unos y otros factores.

- $\mathrm{H}$ ay otros factores de índole social y relacional: conocimiento del entorno, relaciones interpersonales, etc. Las denominadas «redes sociales informales» constituyen uno de los procedimientos más usuales de acceso a un empleo, frente a otras formas más institucionales (IN EM , agencias de selección de personal, etc.). Es en este sentido que se plantea la mayor o menor capacidad de acceso a determinados empleos según el conocimiento del entorno empresarial y laboral. $\mathrm{H}$ ay que subrayar aquí que los colectivos que ocupan los segmentos inferiores, a caballo entre el paro y el sector marginal, parecen entrar en un «proceso de marginalización» (Sengerberger, 1988), que puede implicar un enquistamiento de las personas que los componen en situaciones de difícil reversivilidad (paro de muy larga duración 0 alternancia repetida de paro con empleos precarios e inestables) en relación con su inserción en el mercado de trabajo.

- La capacidad de negociación colectiva es otro factor destacado que influye sobre las posibilidades de acceso a uno u otro segmento y en la movilidad entre los mismos. El grado de asociacionismo, y su incidencia sobre el conjunto de las relaciones laborales, coadyuva a la configuración de puestos de trabajo diferenciados a los que se verán encaminados colectivos concretos $y$, a su vez, distintos.

\section{Análisis de la segmentación del mercado de trabajo}

D e forma coherente con los planteamientos hasta aquí expresados desarrollamos un doble nivel de análisis del trabajo-empleo desde la perspectiva de la segmentación. En primer lugar emplearemos los datos fruto de la explotación de la Enquesta de la Regió M etropolitana de Barcelona de 1990 con objeto de 
elaborar las tipologías de segmentación sobre la base de los criterios señalados. Consecuentemente, surgirán los tipos de trabajo que sobresalen como los más característicos de uno u otro segmento. En segundo lugar configuraremos los grupos sociales que ocupan los diversos segmentos.

A continuación presentamos los resultados del análisis que hemos realizado del ámbito productivo desde el modelo de segmentación. El interés de estas reflexiones no sólo está en los contenidos acerca de la segmentación laboral, sino que también, y de una manera particular, estas reflexiones tienen un interés metodológico, puesto que se han trabajado sobre diversos procedimentos que se han ido afinando y han completado sus resultados progresivamente.

En términos teóricos estrictos la segmentación del empleo es aplicable a los asalariados, puesto que sólo ellos están presentes en el mercado de trabajo. Pero ciertamente los no asalariados, particularmente si son autónomos, pequeños empresarios o trabajan como ayuda familiar, tienen también una posición en el trabajo (hasta el punto de perderlo en ocasiones) y por analogía pueden tener una localización en una teórica escala de segmentación del conjunto de los ocupados. Los parados, por definición, están en el extremo inferior de la escala.

Por estos motivos vamos a establecer inicialmente la tipología de segmentación con variables que se refieren sólo a las condiciones que tienen los asalariados ocupados; pero a continuación agregaremos los dos colectivos señalados: no asalariados ocupados y parados en posiciones de la escala de segmentación que hemos de establecer y justificar en su momento.

Pero si la tipología de la segmentación quiere ser una caracterización en síntesis del trabajo productivo, que pueda ser confrontada con una tipología similar en el trabajo reproductivo y en el ámbito de tiempo libre, hemos de dar cabida en ella a toda la población, aunque en algunos colectivos tal segmentación sea aplicable sólo por analogía. Por tal razón en la tipología final de la segmentación, serán previstos unos segmentos para los inactivos - amas de casas y jubilados/incapacitados - en la forma en que explicaremos posteriormente.

Claro está que no podemos olvidar que, en unos casos, estamos hablando de estructura del mercado de trabajo en términos teóricamente estrictos y, en otros, en términos analógicos y/o agregados. Seguiremos pues en fases progresivas la incorporación de los tres colectivos: ocupados no asalariados, parados, el resto de la población, con vistas a la constitución de una tipología general de estratificación que abarque al conjunto de toda la población.

\subsection{Variables de partida}

Para la construcción de la tipología de segmentación laboral hemos tenido en cuenta diversos factores básicos o dimensiones que razonábamos en la prime-

5. Estos factores o dimensiones no se entienden como independientes y autónomos entre sí, sino que se interrelacionan en el proceso de constitución de la estructura de segmentación. Aś, por ejemplo, la estabilidad del puesto de trabajo, tal como la concebimos aquí, no es sólo de derecho, sino principalmente de hecho 0 , cuando menos, tiene aspectos legales y aspectos 
ra parte de estas páginas ${ }^{5}$. Estos factores centrales que definen los puestos de tra bajo o contextualizan la dinámica del proceso de segmentación del mercado de trabajo se expresan, a través de diversos indicadores, en un conjunto de variables que de forma breve presentamos seguidamente (tabla 1$)^{6}$.

Respecto a la estabilidad del puesto de trabajo, hemos considerado el tipo de contratación laboral que se tiene como el indicador principal de esta dimensión. Esta variable establece la diferencia básica de dos valores: el tener contrato indefinido o de carácter eventual en una de sus diversas modalidades - aquí podemos incluir también la ausencia de contrato- . Esta distinción es fundamental en el contexto del proceso segmentador y es posible acompañarla de otras variables: duración del contrato, tiempo en la empresa actual y tiempo en paro en los últimos años. En segundo lugar, se emplea la categoría profesional como indicador principal de la cualificación ${ }^{7}$. A través de ella se reflejan los niveles de cualificación reconocidos por la empresa mostrando los conocimientos potencialmente requeridos, así como la posición ocupada en la división social del trabajo. Junto a esta cualificación formal cabría contemplar también dos subdimensiones que reflejarían las exigencias o requerimientos del trabajo efectivamente realizado en términos bien de conocimientos (habilidad manual, capacidad intelectual), bien de posibilidades de control del trabajador en la realización efectiva de su propio trabajo (capacidad de intervención, organización y autonomía). Finalmente, un elemento más dinámico de las cualificaciones lo constituiría el hecho de la promoción o no en el trabajo. En tercer lugar, tenemos en cuenta la variable de ingresos expresada en términos de ingresos mensuales netos a precios del año 1989, con información recogida en interval os que ha sido tratada a distintos niveles de agregación.

D entro de las características contextuales consideramos que la posición que se ocupa en el mercado laboral viene condicionada por el hecho de que en la empresa haya canales de representación de los trabajadores - comités y delegados- 0 no existan éstos, por cuanto la existencia de tal representación puededar a muchos trabajadores una capacidad colectiva de mantenimiento del empleo, un poder negociador y unas garantías, que no tendrían individualmente. El tipo de empresa en el que se está trabajando nos permite distinguir entre pública o privada (den-

factuales. Con otras palabras, se puede tener un contrato estable en un sector, en una empresa en crisis, en un puesto de trabajo que desaparezca fácilmente y encontrarse sin la capacidad para reemplazar ese puesto por otro.

6. En la tabla se incluyen las principales variables utilizadas en el análisis, algunas otras que serán citadas no aparecerán por motivos de extensión.

7. La cualificación que refleja la categoría profesional evidentemente no es ajena a la dimensión de estabilidad. Independientemente del tipo de empresa en que se está trabajando, del tamaño de la misma y del contrato que se tenga, se puede tener más o menos capacidad de mantener el puesto de trabajo en función de la categoría profesional. H emos hipotizado que las categorías profesionales más altas de asalariados (técnicos altos, directores o gerentes) tienen hoy día una mayor estabilidad que las categorías intermedias (técnicos medios, personal administrativo o comercial, obreros cualificados), y en mucha mayor medida que las más bajas (trabajadores del comercio y de los servicios, obreros no cualificados). 
tro de éstas últimas es posible distinguir entre sociedades anónimas, cooperativas, familiares y sociedades anónimas laborales). El sector de actividad es susceptible de concretarse en distintas clasificaciones, aquí hemos manejado una de base que distingue: metal, químicas, textil-calzado-piel, electrónica, otras industrias, cons trucción, comercio-hostelería, transportes-comunicaciones, financieras, administración, sanidad, enseñanza y otros servicios. Por fin, el tamaño del centro de trabajo se ha tratado a partir del número de trabajadores, considerando divisiones distintas en torno a la dicotomía de más o menos de veinticinco trabajadores.

Tabla 1. Distribución de frecuencias de las variables del análisis de segmentación del mercado de trabajo.

\begin{tabular}{lrr}
\hline & Efectivos & $\%$ \\
\hline Tipo de contratación & & \\
Sin contrato & 140 & 6,8 \\
Eventual & 596 & 29,1 \\
Indefinido & 1.315 & 64,1 \\
\hline C ategoría profesional & & \\
Obrero no cualificado & 195 & 9,5 \\
O brero cualificado & 446 & 21,8 \\
Trabajador servicios & 473 & 23,1 \\
Contramaestre & 88 & 4,3 \\
Resto administrativos, comerciales y técnicos & 374 & 18,2 \\
Técnico medio & 92 & 4,5 \\
Técnico alto & 383 & 18,7 \\
\hline Ingresos netos mensuales en 1989 & & \\
0-60.000 & 413 & 20,2 \\
60.001-90.000 & 581 & 28,3 \\
90.001-140.000 & 658 & 32,1 \\
M ás de 140.000 & 290 & 14,2 \\
N S/N C & 109 & 5,3 \\
\hline Titularidad de la empresa & & \\
S.A.L./C ooperativa & 148 & 7,2 \\
O tra empresa privada & 831 & 40,5 \\
Sociedad Anónima & 668 & 32,6 \\
Empresa pública-mixta & 404 & 19,7 \\
\hline Tamaño de la empresa (número de trabajadores) & & \\
M enos de 6 & 389 & 19,0 \\
6-25 & 537 & 26,0 \\
26-50 & 289 & 14,1 \\
51-100 & 253 & 12,3 \\
101-500 & 301 & 14,7 \\
M ás de 500 & 285 & 13,9 \\
\hline Tipo de representación laboral en el centro de trabajo & & \\
Comité de empresa & 2454 & 41,7 \\
D elegados de personal & 953 & 11,9 \\
Sin representación & 46,4 \\
\hline Total & 2.051 & 100,0 \\
\hline & & \\
\hline
\end{tabular}




\subsection{La construcción de la tipología de segmentación laboral}

En la construcción de la tipología de segmentación hemos seguido diferentes métodos. Aunque el objetivo fundamental de la investigación se centra, evidentemente, sobre contenidos sustantivos, también perseguimos atestar y comparar diversos procedimientos de métodos y de técnicas. Por ello presentamos distintas propuestas de análisis dando cuenta de su bondad y pertinencia junto a su complementaridad, si es el caso. Los métodos de agrupación de variables en vistas a la construcción de la tipología de segmentación son: técnicas aditivas, ponderadas, de log-lineal y de análisis de correspondencias/clasificación. En los tres primeros utilizaremos un conjunto más reducido de variables, trabajamos con cinco variables - tipo de contrato, categoría profesional, representación laboral, tamaño del centro de trabajo y tipo de empresa- que además se han dicotomizado por diversas razones: exigencias de la técnica, finalidad reductora, pertinencia de los ítems o sencillez del proceso ${ }^{8}$.

\subsubsection{La segmentación por una tipología aditiva}

Esta forma de construcción de la tipología de segmentación la podemos cata logar de simple. Se construye dando igual peso a cada una de las variables que entran en el espacio de atributos que la define, y los valores que se obtienen son el resultado de sumar los valores de todas las variables. $D$ ado que las variables son 5 y que éstas están dicotomizadas con valores 0 y 1, los valores de la escala de segmentos inferiores a segmentos superiores irán de 0 a 5. Los resultados obtenidos tras la oportuna agrupación son los siguientes:

\begin{tabular}{rr|r|r|r}
\hline Segmento inferior & 0 & $17,0 \%$ & $17,0 \%$ & \multirow{2}{*}{$59,3 \%$} \\
\hline & 1 & 21,1 & \multirow{2}{*}{$42,3 \%$} & \\
\hline & 2 & $21,2 \%$ & & \multirow{2}{*}{$40,7 \%$} \\
\hline & 3 & $27,3 \%$ & $38,1 \%$ & \\
\hline Segmento superior & 4 & $10,8 \%$ & $2,6 \%$ & \\
\hline
\end{tabular}

Esta tipología nos pone de manifiesto que la proporción de asalariados que gozan de condiciones superiores a la media de los segmentos es menor que la proporción de los que están por debajo. H emos contrastado además esta tipo-

8. La codificación a dos valores de estas cinco variables se expresa en los siguientes términos, con las frecuencias relativas de cada categoría:

\begin{tabular}{lllll}
\hline Tipo de contrato: & 0 no fijo & $(35,9 \%)$ & 1 fijo & $(64,1 \%)$ \\
Categoría profesional: & 0 no técnicos & $(76,8 \%)$ & 1 técnicos & $(23,2 \%)$ \\
Representación laboral: & 0 no representación & $(46,4 \%)$ & 1 representación & $(53,6 \%)$ \\
Tamaño de la empresa: & 0 pequeña & $(45,0 \%)$ & 1 grande & $(55,0 \%)$ \\
Tipo de empresa: & 0 privada & $(80,3 \%)$ & 1 pública & $(19,7 \%)$ \\
\hline
\end{tabular}


logía con cada una de las variables que la componen, con el fin de obtener una primera aproximación sobre su consistencia. Esta primera aproximación nos permite señalar, como no podía ser menos, que los segmentos superiores se dan en la empresa pública y grande y, contrariamente, los segmentos inferiores, en la empresa privada y pequeña. En los segmentos inferiores se acentúa el hecho de que en la empresa no haya representación de los trabajadores. Así mismo, los segmentos más sólidos se dan cuanto mayor es la presencia de trabajadores con categoría de técnicos. Por último, se da máxima estabilidad con contrato fijo y mínima con contrato eventual o sin contrato. Todas estas asociaciones son altamente significativas, lo que pone de manifiesto que la tipología de estabilidad es mucho más consistente que cada una de sus componentes.

\subsubsection{La segmentación por una tipología ponderada}

La perspectiva teórica de la segmentación, avalada por diversas investigaciones, ha subrayado la importancia central de los factores o dimensiones que definen la estabilidad y la cualificación. Por eso hemos querido experimentar otra tipología de segmentación que atribuyera pesos diferenciados a los valores de las cinco variables analizadas, concretamente los siguientes: un peso 3 a los valores del tipo de contrato y la categoría profesional; un peso 2 a la variable tipo de empresa y representación laboral, y un peso 1 al tamaño de la empresa.

El objetivo de esta ponderación es doble: acentuar la importancia de dos factores que parecen claves en la idea de segmentación laboral y diluir, en cierto modo, el peso preponderante del tamaño de empresa. Como es lógico esta nueva tipología comporta 12 valores, siendo el mínimo 0 y el máximo 11 (la suma de todos los máximos posibles). Los resultados son los siguientes:

\begin{tabular}{|c|c|c|c|c|}
\hline Segmento inferior & 0 & $17,0 \%$ & & \multirow{6}{*}{$59,2 \%$} \\
\hline & 1 & $4,8 \%$ & $21,8 \%$ & \\
\hline & 2 & $2,5 \%$ & \multirow{4}{*}{$37,4 \%$} & \\
\hline & 3 & $21,1 \%$ & & \\
\hline & 4 & $6,6 \%$ & & \\
\hline & 5 & $7,2 \%$ & & \\
\hline & 6 & $23,5 \%$ & \multirow{4}{*}{$37,2 \%$} & \multirow{6}{*}{$40,8 \%$} \\
\hline & 7 & $2,2 \%$ & & \\
\hline & 8 & $7,1 \%$ & & \\
\hline & 9 & $4,4 \%$ & & \\
\hline & 10 & $1,0 \%$ & \multirow{2}{*}{$3,6 \%$} & \\
\hline Segmento superior & 11 & $2,6 \%$ & & \\
\hline
\end{tabular}

El primero y el último valor, segmentos inferior y superior, coinciden en ambas tipologías. Si dividimos ambas tipologías en cuatro bloques - segmentos inferiores, segmentos medios inferiores, segmentos medios superiores y seg- 
mentos superiores- podemos observar que la tipología ponderada acrecienta los casos en la zona más inestable a costa de la zona media-baja, mientras que la alta y la media alta queda inalterable. Es decir, al dar mayor peso a dos variables una de las cuales es la categoría profesional se distribuye algo más deshomogéneamente la población acentuando el peso de los segmentos inferiores. Pero como en la precedente tipología se pone de manifiesto la proporción de asalariados que gozan de condiciones superiores a la media de la tipología es menor que la proporción de los que están por debajo.

\subsubsection{La segmentación por modelos empíri cos de asociación dados por método de log-lineal}

El siguiente paso realizado ha consistido en examinar, previamente a la construcción de cualquier tipología, el modelo de asociación entre el conjunto de las variables. El análisis log-lineal es un método cuyo objetivo consiste en identificar o validar modelos de relaciones de asociación entre variables de tipo cualitativo, además de permitir conocer las influencias parciales de los valores de las variables o de sus asociaciones en la creación de las frecuencias esperadas por el modelo. Para ello tranfforma las relaciones entre valores de las variables cualitativas en forma de relación lineal. Así pues, el método posibilita ajustar el modelo global y localmente. Cuando se trabaja con muchas variables los model os posibles se complican considerablemente, pues las mutuas dependencias globales y locales se multiplican. El tratamiento log-lineal en este caso tiene como objetivo el de indagar e identificar el modelo de asociación entre variables que más se ajusta a los datos empíricos, a fin de que sea utilizado como un criterio de ponderación de las diferentes variables en vistas a la construcción de la tipología de segmentación que buscamos. No entraremos pues en el análisis de los parámetros que nos dan el valor y la significación de las asociaciones locales.

El modelo de asociación global resultante destinado a la construcción de la tipología de la segmentación laboral es el resultado de la utilización de las variables hasta ahora consideradas en el análisis en su expresión dicotómica en vistas a la simplificacion recomendable del log-lineal. Este modelo es el resultado también de la revisión de algún ejercicio anterior. En concreto habíamos considerado en un inicio las cinco variables dicotomizadas donde se obtenía una tipología con el inconveniente que introducía el peso que se otorga a la categoría profesional, lo que nos ha conducido a introducir alguna variación en su definición. Consideramos técnicos o cualificados (frente a no técnicos o no cualificados), con valor 1 frente a valor 0 , a aquellos que por categoría son técnicos más aquellos que declaran que han visto incrementado el uso de conocimientos en su trabajo en el último período; esto nos permite captar a asalariados no técnicos cuyo trabajo tiene un contenido rico en cualificación. En este caso el reparto de frecuencias es $42 \%$ para no técnicos y $58 \%$ para técnicos.

Según los resultados del log-lineal el modelo de asociación que se ajusta a los datos empíricos es el representado gráficamente de la forma: 


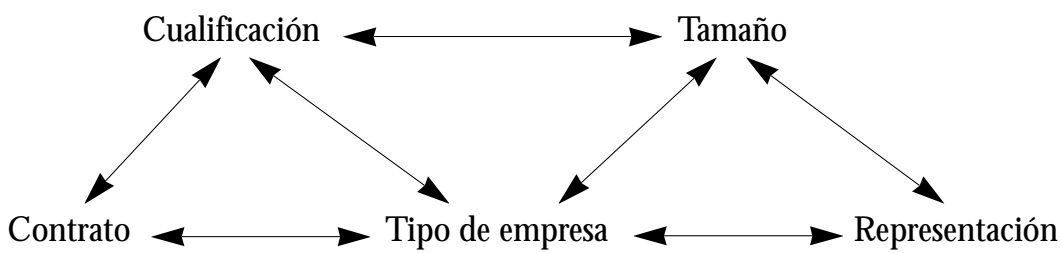

Este model o estructura la relación entre las cinco variables. Consiste en un modelo de triple asociación trifactorial o tres de triple efecto formadas por los tres triángulos correspondientes. No aparecen efectos de cuarto o quinto orden. Se trata de utilizar este modelo como un criterio para ponderar adecuadamente cada variable en vistas a la creación de la tipología de segmentación. La máxima asociación la tenemos entre las variables Tamaño, Tipo de empresa y Representación que se constituye como el eje de más fuerza de orden triple. A continuación tenemos la asociación entre Cualificación, Tipo de empresa y Tamaño. Por último se configura el triángulo Tipo de contrato, Cualificación y Tipo de empresa.

Como se puede observar, el Tipo de empresa es el punto sobre el que se apoyan las tres asociaciones trifactoriales, por tanto quien mejor se asocia con el conjunto de los factores considerados en la construcción del modelo de segmentación, es el centro sobre el que se apoyan las demás variables. Las razones de tal centralidad se encuentran en que se trata de una variable respecto a la cual más «netamente» se sitúan los valores de las demás variables, es decir, la empresa pública es claramente empresa grande y donde es mayoritario el trabajador técnico; también aquélla en la que la contratación es más fija y aquella que más fácilmente puede contar con representación laboral. Situación que no se da con la misma intensidad en la empresa privada.

Estos resultados obtenidos podemos emplearlos como criterios de construcción de una tipología de segmentación tendente a dimensionalizar las relaciones entre variables y clasificar a la población asalariada. D e esta forma podemos distinguir en primer lugar la existencia de tres ejes asociativos:

1) El primer eje asociativo o dimensión contextual. De las asociaciones triples, la que se conforma con Tamaño, Ti ipo de empresa y Representación es la más fuerte. Por tanto, ésta se configura como el eje básico de la asociación global. En cierto modo son variables propias y genéricas de la empresa, del puesto de trabajo e independientes del individuo. A pesar de tener la máxima asociación, las variables de este eje no todas tienen una fuerte relación con el conjunto de las cinco. Por el contrario, la Representación queda desvinculada de las otras variables, mientras que el Tamaño y el Ti po de empre sa están asociados ambos a la Cualificación, y también existe una relación entre Tipo de empresa y Contrato.

D ada la fuerza asociativa de este primer eje en la estructura asociativa de las cinco variables, daremos una mayor ponderación a ellas en la creación 
de la tipología de segmentación laboral, con una propuesta de construcción y distribución de valores del eje reducida a una expresión dicotómica resultado de la agrupación de las distintas combinaciones de valores de las variables originales:

\begin{tabular}{cccc}
\hline Tamaño & Representación & Tipo de empresa & Eje 1 \\
\hline 1 & 1 & 1 & \\
1 & 1 & 0 & Eje $1=1$ \\
1 & 0 & 1 & \\
0 & 1 & 1 & \\
1 & 0 & 0 & \\
0 & 1 & 0 & Eje $1=2$ \\
0 & 0 & 1 & \\
0 & 0 & 0 & \\
\hline
\end{tabular}

2) EI segundo eje asociativo o de cualificación. Este segundo eje en el que entran las variables Tipo de empresa, Tamaño y Cualificación ocupa una posición central en el modelo de asociación, pues sus tres variables tienen relación con el resto. Contiene variables, en parte pertenecientes a la empre sa en parte inherentes a la capacidad propia del asalariado. Es una dimensión de la segmentación que relaciona al individuo con la empresa (o el puesto) y, al mismo tiempo, a partir de las capacidades aportadas por él principalmente a través de la formación adquirida, por más que está claro que también en la empresa se adquiere dicha formación o cualificación. Con otras palabras, el tener una determinada cualificación posibilita entrar 0 mantenerse en la empresa pública y grande, siendo esto fundamental para la estabilidad y siéndolo menos el tipo de contrato que se tenga, si bien también existe una relación de asociación de contratación indefinida y empresa pública que se pondrá de manifiesto en el tercer eje. Podemos llamar a este segundo como eje de cualificación. Es un eje que opera conectado con el anterior a través de uno de sus tres lados y podemos expresarlo con las siguientes combinaciones de valores y frecuencias:

\begin{tabular}{cccc}
\hline Tamaño & Cualificación & Tipo de empresa & Eje 2 \\
\hline 1 & 1 & 1 & \\
1 & 1 & 0 & Eje 2 $=1$ \\
1 & 0 & 1 & \\
0 & 1 & 1 & \\
0 & 1 & 0 & \\
1 & 0 & 0 & \\
0 & 0 & 1 & Eje 2 $=2$ \\
0 & 0 & 0 & \\
\hline
\end{tabular}


3) El tercer eje asociativo o de estabilidad contractual. En este caso la triple asociación se da entre las variables Tipo de empresa, Contrato y Cualificación. Es el eje que presenta la interacción más débil entre sus variables y, al mismo tiempo, el que se encuentra más desplazado del conjunto de la interacción. Como adelantábamos anteriormente, es el eje que pone de manifiesto la vinculación de la contratación indefinida y la alta cualificación como características propias o más destacadas de la empresa pública. En este caso podemos resumir esta información en términos del siguiente cuadro:

\begin{tabular}{cccc}
\hline Contrato & C ualificación & Tipo de empresa & Eje 3 \\
\hline 1 & 1 & 1 & \\
1 & 1 & 0 & Eje 3 =1 \\
1 & 0 & 1 & \\
0 & 1 & 1 & \\
1 & 0 & 0 & \\
0 & 1 & 0 & Eje 3 $=2$ \\
0 & 0 & 1 & \\
0 & 0 & 0 & \\
\hline
\end{tabular}

Tenemos pues tres dimensiones o ejes de dimensionalización de la segmentación que no hay que entenderlos como independientes entre sí, ya que tienen buena parte en común puesto que provienen de variables compartidas aparte de la propia asociación. Los tomamos en grupos de asociación entre tres según el modelo (podría haber habido efectos de orden cuatro o más), donde las diferencias entre ellos provienen, como hemos visto, de a lo sumo una variable según lo obtenido del modelo de asociación validado. Ahora podemos construir, en segundo término, una tipología de segmentación única que se obtiene por combinación de todos los posibles valores de los tres ejes que hemos descrito - un total de ocho combinaciones o tipos- , que a su vez es reagrupada en cuatro val ores. O btenemos los siguientes tipos o segmentos que reflejan la variabilidad del segmento inferior al superior:

$\left.\begin{array}{ll}\text { Segmento } 1 & 33,6 \% \\ \text { Segmento } 2 & 15,0 \% \\ \text { Segmento } 3 & 18,4 \% \\ \text { Segmento } 4 & 33,0 \%\end{array}\right\} 48,6 \%$

Comparando esta segmentación con las precedentes vemos que equilibra los valores superiores e inferiores a una media de segmentación. Esta tipología, estratificación de asalariados, mantiene una asociación al ta con cada una 
de las variables que la constituyen ${ }^{9}$. Veamos ahora el contenido de los tipos de segmentación laboral. Este contenido lo identificaremos primero a partir de las variables que los constituyen y luego se dará una caracterización de cada tipo a partir de un conjunto de variables de identificación social, esto nos permitirá saber a qué empleos nos referimos cuando hablamos de segmentación y nos dará la base para, seguidamente, describir los grupos social es que corresponden a cada segmento. Este contenido social nos permitirá con posterioridad realizar la comparación con los otros ámbitos - reproductivo y de tiempo libre- que estudiamos en la investigación.

- Segmento 1: segmento inferior. Se concentra totalmente en la empresa privada, en un $86 \%$ están en empresa pequeña, en casi el $90 \%$ de los casos no tiene representación laboral, en tres cuartas partes se trata de no cualificados, en más del $70 \%$ son eventuales o sin contrato.

- Segmento 2: segmento medio-bajo. Están totalmente en la empresa privada, en casi un $80 \%$ y en empresa pequeña, en tres cuartas partes sin representación laboral, sin embargo, son en su totalidad cualificados y en casi un $80 \%$ de contrato fijo. En el grado en que la cualificación tiene un peso consistente en la tipología construida, podríamos pensar que la debilidad estructural queda corregida. $\mathrm{H}$ ay base para pensar que se trata de un segmento en el que un grupo de personas están en período detransición hacia el segmento superior.

- Segmento 3: segmento medio-alto. Están en un $90 \%$ en la empresa privada, y en idéntica proporción en la empresa grande, teniendo en un $67 \%$ representación laboral; son profesionalmente poco cualificados en un $67 \%$, y con una estabilidad contractual que lleva a que los fijos representen las tres cuartas partes.

- Segmento 4: segmento superior. M ás del 85\% de estos empleos están en la empresa pública y en casi un $95 \%$ en la empresa grande, tienen en más del $90 \%$ de los casos representación laboral, son cualificados en un $88 \%$ y fijos en más de tres cuartas partes.

Los tipos o estratos extremos son los que en todas las formas de contrastación aparecen con diferencias más nítidas frente a una menor claridad de los tipos intermedios. No conviene olvidar que los estratos extremos abarcan casi el $67 \%$ de la población asalariada. Respecto a los ejes de los que ha partido la estratificación que estamos analizando, parece clara la incidencia del primer eje - en positivo o en negativo- sobre el conjunto de los individuos; el segundo eje tiene un papel relevante - en positivo o en negativo- principalmente en los tipos intermedios, por el contrario, el tercer eje tiene escaso papel:

9. Por ejemplo, en el coeficiente de asociación Phi es el siguiente para cada variable: Tipo de empresa: 0,50; Representación: 0,68; Cualificación: 0,66; Contrato: 0,47; Tamaño: 0,78, evidentemente significativos en todos los casos. La asociación es también muy fuerte con las dos primeras tipologías construidas. 
podríamos decir que acentúa la inestabilidad del primer tipo y consolida la estabilidad del cuarto.

Veamos ahora la identidad social de estos segmentos a partir de una serie de factores contextuales y estructurales. Esto nos permitirá descubrir un grupo 0 unos grupos sociales en cada segmento que están compuestos por las personas que tienen más probabilidad de ir a parar a los diversos segmentos ${ }^{10}$.

El segmento 1 lo componen trabajadores con salarios mayoritariamente bajos y jornada corta (aunque también hay un subgrupo con jornada más larga que la normal); tienden a ser jóvenes (aunque también los hay de otras edades, al menos en 1/4 de la población de todas ellas); se trata sobre todo de mujeres, sin que el tipo de familia sea muy discriminante; de renta familiar baja o desconocida; de categoría familiar baja, sin estudios o con estudios primarios. En este estrato se encuentran los inestables con menos recursos personal es y, probablemente, mas «cautivos» en esa situación marginal.

En el segmento 2 entran con mayor probabilidad personas con salarios mediosal tos y jornada alta. Suelen tener entre veintiséis y cuarenta y cinco años, en mayor proporción hombres que mujeres; no es significativo el tipo de hogar; de renta familiar media-alta; de categoría socioeconómica alta y media; de estudios secundarios y universitarios. Aparecen personas que en algún grado no son estables, pero por lo que se ve tienen recursos personales para aguantar esa situación hasta encontrar al go mejor. La edad y el tipo de familia del que provienen (ingresos, categoría familiar) avalan la hipótesis de estrato de transición al cuatro, como señalábamos anteriormente.

Al segmento 3 van con mayor probabilidad quienes tienen salarios medios y jornadas medias. Son personas de edad madura, en mayor proporción hombres que mujeres, de familias unipersonales más que de otro tipo, con renta familiar de todos los niveles excepto el alto, de categoría baja y media, sin estudios o con estudios primarios, nacidos fuera de C ataluña y de zonas periféricas a Barcelona. Algunos de estos factores son claros indicadores de inseguridad, particularmente si comparamos este estrato con el siguiente. La diferencia fundamental entre este tipo y el anterior está en que en éste las personas tienen puestos de trabajo objetivamente más estables pero tienen menos recursos personales en términos de estudios, renta familiar, categoría socioeconómica, etc., además de que son mayoritariamente inmigrantes y residentes en las comar-

10. Las variables sociales con las que han sido contrastados estos tipos aparecen a continuación, entre paréntesis aparece el valor del coeficiente de asociación Phi y el nivel de significación: Ingresos (0.41 / 0.000), J ornada laboral (0.23 / 0.000), Edad $(0.26$ / 0.000), Sexo (0.17 / 0.000), Tipo de familia (0.12 / 0.028), Renta familiar ( 0.29 / 0.000), Categoría socioeconómica $(0.36 / 0.000)$, Nivel de estudios acabados $(0.29$ / 0.000$)$, O rigen geográfico $(0.12$ / 0.0009), Tamaño del municipio $(0.09$ / 0.072), División del territorio por coronas $(0.09$ / 0.004), División del territorio por subcoronas $(0.15 / 0.000)$. Los niveles de asociación son aceptables salvo con las variables de origen y residencia. 
cas externas a Barcelona frente a los del 2, que son mayormente nacidos en $C$ ataluña y residentes en Barcelona.

Finalmente, en el segmento 4 se hallan con mayor probabilidad asalariados con salarios al tos y jornadas medias. Están en las edades centrales - de veintisés a cincuenta y cinco años- , con mayor probabilidad hombres que mujeres aunque las diferencias no son excesivamente exageradas; sobresalen los hogares unipersonales, de renta familiar al ta y media-alta, de categoría familiar alta, de estudios universitarios; son más bien de Barcel ona que de las otras comarcas, pero sin que esta diferencia sea excesiva. N os hallamos ante el tercio de la población segura, estable y con trabajo garantizado.

\subsubsection{La segmentación por una tipología estructural y articulada}

Este análisis no pretende suplantar la tipología conseguida hasta ahora, que como vemos es rica en resultados. Si aplicamos diversos métodos es guiados por el interés de que esta investigación no sólo contenga elementos sustantivos, sino que sea también un análisis de comparación entre métodos. El procedimiento de construcción que ahora proponemos es una extensión y una complementación de los vistos hasta ahora que nos permitirá trabajar con la información más desagregada - no con valores dicotomizados- y con un número mayor de variables. D e esta forma pretendemos como objetivo tener en cuenta un modelo de interrelación más complejo y enriquecedor de los resultados del análisis de estructuración del fenómeno de la segmentación bajo una forma tipológica. La forma de construcción además sigue un procedimiento metodológico que lleva a calificar a la tipología así obtenida como estructural y articulada11.

La información que se maneja parte de la caracterización que se realiza de la población asalariada según el conjunto de catorce variables que definen el espacio de atributos de estructuración del mercado de trabajo. Estas variables surgen de la distinción de cuatro dimensiones principales con las que ahora caracterizamos el empleo desde la perspectiva de la segmentación, según comentábamos más arriba.

En relación con diseño de análisis, comentar tan sólo que éste se organiza a partir de la distinción de dos momentos principales, sucesivos, complementarios y recurrentes: en primer lugar, una etapa de dimensionalización destinada a establecer una estructura inicial del espacio de atributos que delimita la tipología en el campo conceptual que hemos comentado por medio de la combinación y la reducción de las variables originales (mediante la técnica del análisis de correspondencias múltiples). En segundo lugar, una etapa de clasi-

11. Los comentarios que siguen recogen parte de un trabajo que ya ha sido publicado (P. López, 1994, 1996b). Sobre el concepto de tipología estructural y articulada se puede consultar P. López (1994, 1996a), sobre el diseño de análisis ver M. D omínguez y P. López (1996). O tros ejemplos de construcción tipológica bajo esta concepción se pueden seguir en el numero monográfico 48 de la revista PAPERS, correspondiente al año 1996. 
ficación destinada a constituir los tipos de la tipología como conjunto reducido de categorías (mediante diversas técnicas de clasificación automática). A continuación se presentan los principales resultados a los que nos conduce el proceso de construcción y validación de la tipología de segmentación del empleo.

El análisis de estructuración inicial mediante el análisis de correspondencias múltiples nos soluciona el problema de cómo establecer, combinar y reducir el espacio original de catorce variables - y cincuenta y dos categorías 0 modalidades asociadas- a unos pocos factores o dimensiones que expliquen y estructuren las situaciones del empleo asalariado. D e este análisis resultan cinco variables factoriales que caracterizan de forma jerárquica a la población asal ariada de la siguiente forma:

La primera dimensión obtenida corresponde a la oposición etabilidad-inestabilidad laboral, introduciendo la distinción entre los rasgos característicos del empleo primario y secundario. Se trata de un primer y principal factor que expresa la dualización del empleo asalariado. La segunda dimensión que se obtiene se identifica fundamentalmente en términos de cualificación y diferencia un perfil más cualificado propio del segmento primario independiente y otro de menor cualificación propio de un segmento primario dependiente. Consiste pues en un factor de diferenciación y jerarquización del empleo estable que surgía en la primera dimensión. Junto a esta distinción se establece otra respecto al empleo más inestable: entre los asalariados que trabajan de forma sumergida y los eventuales de reciente incorporación al mercado laboral.

Junto a estos dos factores centrales que estructuran las diferencias entre la población asalariada, los análisis efectuados nos conducen a considerar tres más, de menor importancia, pero que ayudan a matizar las principales relaciones que dibujan la estabilidad y la cualificación. La tercera dimensión se identifica con relación a la titularidad de la empresa, pero oponiendo el empleo de menor cualificación efectiva de la gran empresa pública frente al empleo más enriquecedor de categorías profesionales intermedias de la pequeña empresa privada. La cuarta dimensión se puede calificar de continuidad-discontinuidad laboral, pues nos segrega trayectorias marcadas por la eventualidad, con la entrada y salida del mercado de trabajo, de la permanencia en la ocupación y en la misma empresa. Por último, en la quinta dimensión toma peso la categoría profesional para distinguir fundamentalmente el empleo público entre técnicos al tos y trabajadores de servicios.

Una vez establecidos estos cincos factores de heterogeneidad de las situaciones de empleo, ahora se trata de conseguir aquellos grupos o clases que a efectos de estructuración del fenómeno de la segmentación den lugar a un conjunto de tipos o segmentos de empleo lo más homogéneos internamente, por tanto, lo más heterogéneos entre ellos, según las variables criterio o dimensiones de la segmentación. Tras el proceso de validación técnica y teórica de los resultados obtenidos, el contenido final de las clases o grupos resultantes se expresa en términos de cuatro tipos de la tipología que permiten estructurar la realidad del empleo en la Región M etropolitana de Barcelona desde la pers- 


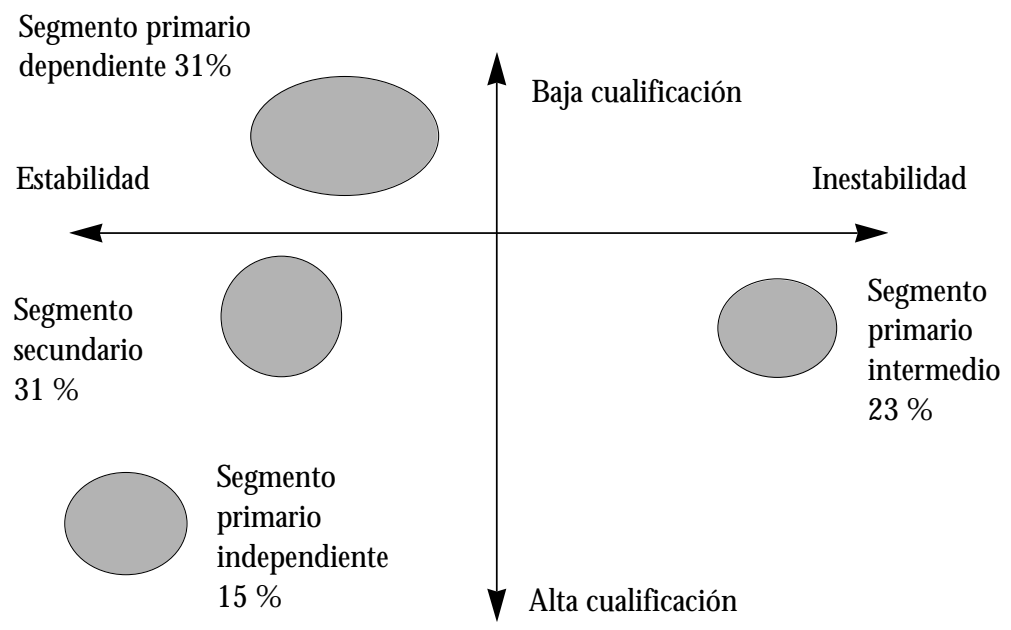

\section{Gráfico 1}

pectiva de la segmentación. Tres de estos tipos se corresponden con la caracterización del segmento primario, mientras que el cuarto responde a la identificación del segmento secundario. En el gráfico adjunto (gráfico 1) se reflejan estos cuatro tipos por su ubicación en los dos principales ejes factoriales 0 dimensiones de la segmentación. A continuación describiremos el contenido de los segmentos de empleo resultantes.

El primer tipo (31\%) es el que denominamos como segmento primario dependiente. Contiene las situaciones de empleo estable y corresponde a personas que en su mayoría llevan más de quince años en la empresa y casi todos más de cinco. Aquí confluyen otras dos características definitorias. Por un lado, se trata del tipo de empleo estable con una menor cualificación. Está constituido por asalariados de categorías profesionales medias y bajas, fundamentalmente de obreros cualificados y no cualificados y trabajadores de servicios, que manifiestan un estancamiento profesional derivado de no haber ascendido de categoría en los últimos años, con una mínima capacidad de control sobre su propio trabajo. M ayoritariamente ocupan puestos de trabajo a los que se asocian exigencias como la precisión en el cumplimiento de las normas y la obediencia, desarrollando capacidades de habilidad manual y resistencia física. C on todo, la variación de los conocimientos técnicos no adquiere una valoración tan negativa, si bien persiste una mayoría que considera que estos conocimientos no han aumentado en los últimos cinco años. Esta moderación del aspecto de los conocimientos en la cualificación confirma la dinámica propia de un perfil general que se identifica plenamente con la caracterización del segmento primario dependiente, donde en particular se ha destacado el proceso de adquisición de cualificaciones más o menos específicas como resultado de la formación y el aprendizaje en el puesto de trabajo. 
Por otro lado, se trata del tipo de empleo que se da en mayor proporción en la empresa mediana y, sobre todo, en la gran empresa. Paralelamente se registran los mayores niveles de representación laboral de los trabajadores, lo que permite reforzar las garantías de estabilidad laboral de un empleo de más baja cualificación. Como se trata de un tipo de empleo coincidente con el que se ha tipificado habitualmente un segmento del sector industrial, aquí podemos constatar cómo se localiza tanto en subsectores de la industria como de los servicios. En particular cabe destacar la presencia diversificada de distintos subsectores, pero sobresalen en mayor medida el metal, químicas, textil/calzado/piel, otras industrias, transportes/comunicaciones y administración, permitiendo mantener la distribución global de los asalariados entre empresas privadas y públicas.

La caracterización de este tipo o segmento de empleo se completa con una distribución de los ingresos mensuales netos concentrado en los niveles intermedios, entre las 60 y las 140 mil ptas. Por otra parte, si atendemos al perfil social de este colectivo, se puede comprobar el predominio de las edades más adultas, por encima de los cuarenta años de edad, con una mayoría de varones que al canza el $73 \%$ del total de este primer tipo. El nivel de estudios acabados es el más bajo de todos los asalariados, el $68 \%$ no supera los estudios primarios. El resto de las variables ilustrativas que hemos considerado nos indican la presencia mayoritaria de personas nacidas fuera de Cataluña, que no emplean la lengua catalana, coincidiendo con los procesos inmigratorios que tuvieron lugar en los años sesenta.

Frente a esta definición descriptiva del primer tipo, el segundo (23\%), identificado como segmento primario intermedio, representa una situación de empleo estable e intermedia entre el tipo anterior y el que describiremos a continuación. Intermedio también por su comportamiento en las distintas dimensiones con las que hemos estructurado el espacio de atributos del fenómeno de la segmentación. D esde el punto de vista de la estabilidad, además de recoger el empleo basado en relaciones contractuales de carácter indefinido y de personas que no han estado desempleadas en los últimos años, este tipo se caracteriza por una vinculación a la empresa menos prolongada en el tiempo de la que aparecía en el primero, concentrándose sobre todo en el período que va de los cinco a los quince años, al mismo tiempo que incluye una pequeña parte del total de empleo eventual, un 10\%, que lleva más de dos años en la empresa y que guarda las mismas características que describimos seguidamente.

Se trata, en primer lugar, de un tipo que coincide con el primero en la presencia de categorías profesionales intermedias y bajas, pero sobre todo de obreros cualificados, contramaestres y resto de personal administrativo, comercial o técnico, con una proporción también, aunque reducida, de técnicos medios y altos. Esta superior cualificación profesional se ve acompañada por la realización de trabajos que exigen primordialmente iniciativa y conocimientos, así como capacidad de organización. Además, para los empleados que ocupan esos puestos de trabajo ha supuesto, con relación a años anteriores, un proceso de promoción profesional (el $67 \%$ ha ascendido de categoría), y para práctica- 
mente la totalidad de ellos, más posibilidades de intervención y de aplicación de conocimientos técnicos.

En segundo lugar, el rasgo que particulariza la naturaleza de este empleo más cualificado es la presencia mayoritaria de la pequeña empresa, hasta veinticinco trabajadores, y en segundo término la de tamaño medio, hasta cien, todas ellas de titularidad privada. Este predominio de pequeña empresa se acompaña también de una ausencia de instancias de representación de los trabajadores que alcanza el $64 \%$ de los empleos de este tipo. Esta realidad laboral de la natural eza de las empresas se completa con una distribución de subsectores de actividad que equilibra la distinción entre industria y servicios, pero donde destacan especialmente los del metal, otras industrias, comercio/hostel ería y financieras/servicios a las empresas.

Por último cabe señalar que la distribución de los ingresos de este tipo supera en promedio a los del primero por la mayor proporción de ingresos altos, por encima de las 140 mil ptas. mensuales, aunque mayoritariamente también se concentra en el intervalo de las 60 a 140 mil. Comparando este segundo tipo con el primero respecto a las variables de identificación social se aprecia la coincidencia en el predominio de los varones, pero no aś en la distribución de edades, donde, si bien aparecen efectivos de todas ellas, las mayores proporciones se encuentran entre los veinticinco y treinta y nueve años. D iferente también es el nivel educativo de este colectivo, con un comportamiento similar al del conjunto de los asalariados y que tiende a acentuar los estudios intermedios (formación profesional y bachillerato). La mayor juventud determina a su vez una mayor presencia de nacidos en Cataluña que el primer tipo, y similar a la de los otros dos que comentaremos. Sin embargo, tanto este segundo tipo como sobre todo el tercero, son los que muestran una mayor familiaridad con la lengua catalana, que contrasta con el comportamiento los tipos primero y cuarto.

La consideración del tercer tipo (15\%), el segmento primario independiente, completa la segmentación del empleo más estable y de perfil identificativo del segmento primario que pemite establecer una ordenación de las situaciones de empleo que culmina en su nivel superior este tercero. En efecto, se trata del empleo que reúne las mejores condiciones según la eval uación derivada de las distintas dimensiones factoriales.

Coincidiendo con los dos anteriores en la estabilidad del empleo que significa la contratación indefinida, destaca sobre todo la continuidad laboral asociada a llevar de cinco a quince años en la empresa y no haber estado desempleado en los últimos años. También aquí, como sucedía con el segundo tipo, se incluye una pequeña parte del empleo eventual, el $9 \%$ del mismo, que verifica de forma acentuada el perfil de este tercer tipo.

El aspecto más característico que lo identifica es el de la cualificación efectiva, y el hecho de que el $84 \%$ se corresponde con la categoría profesional de técnico superior. C onsecuentemente, las exigencias relacionadas con este tipo de empleo son de forma predominante las más autónomas, aquéllas que requieren iniciativa propia, creatividad o conocimientos. Con semejante intensidad, la experiencia laboral reciente de estos asalariados implica más 
posibilidades de intervención como de utilización de conocimientos técnicos. Sin embargo, respecto a la promoción laboral, por corresponderse con profesionales que llevan tiempo en la empresa ocupando los niveles superiores de la escala laboral, presentan una baja proporción de personas que han ascendido de categoría, sobre todo si se compara con el comportamiento del segundo tipo.

0 tro de los aspectos definitorios de este grupo está dado por la combinación de características de las empresas donde trabajan. Se trata sobre todo de asalariados del sector público, el 67\% está empleado en este tipo de empresa, y conjuntamente con los asal ariados de las empresas de titularidad privada concentran su presencia en tres subsectores: enseñanza, administración y sanidad. Esta circunstancia determina la mayor proporción de centros de trabajo de gran tamaño, pero donde también aparecen pequeñas y medianas empresas, siendo globlamente un rasgo común la existencia de representación laboral bajo la forma de comités o delegados de personal.

Las mayores garantías y mejores condiciones laborales que se dibujan en este tipo se traducen también en la más alta remuneración, en niveles que sobrepasan las 90 mil ptas. mensuales a precios del año 1989. Su caracterización social da lugar a una distribución de edades similar a la del segundo tipo, es decir, con edades situadas entre los veinticinco y los cuarenta y cuatro años. Pero, a diferencia de él, aquí son mayoritarias las mujeres, en una relación con los varones que se reparte entre el 54 y el 46 por ciento, y que se explica por la doble confluencia de empleo público y de subsectores con una importante presencia femenina. Como indicamos anteriormente, se trata también de un tipo con personas nacidas sobre todo en $\mathrm{C}$ ataluña, siendo el que en mayor medida utiliza el catalán como lengua habitual y donde se alcanzan los mayores niveles educativos (el $70 \%$ tiene estudios superiores).

Finalmente, la tipología obtenida de segmentos de empleo se completa con el cuarto tipo (31\%), el segmento secundario, que se opone a todos los anteriores por la dualidad que introduce la primera dimensión de segmentación. En este tipo nos encontramos dos realidades del empleo que se aproximan por los acentuados rasgos de precariedad laboral: el empleo estrictamente eventual y el empleo sumergido. Si bien esta distinción en el interior de este tipo se asocia con ciertos rasgos de diferenciación, constituyen características menos relevantes en una partición en cuatro tipos que las que marca la separación de un segmento primario o central, con sus diferencias internas, y un segmento secundario de empleo inestable.

D e esta forma la definición y especificidad de este tipo viene enfatizada por la inestabilidad que se refleja en las distintas modalidades de situaciones y trayectorias de eventualidad laboral, con excepción de una pequeña proporción de este empleo que se incluye en tipos anteriores. A la eventualidad se vinculan otros rasgos como la baja cualificación efectiva, los bajos ingresos y el predominio de la pequeña empresa privada.

Se trata de un colectivo de asalariados con categorías profesionales medias y, sobre todo, bajas, donde destacan los trabajadores de servicios. Entre los distintos 
componentes que completan el concepto de cualificación efectiva priman aquí, en correspondencia con lo que sucede con el primer tipo menos cualificado y de forma algo más acentuada, la experiencia laboral y la realización de tareas marcadas por la ausencia de procesos de recualificación y control del propio trabajo. Así se pone de manifiesto al observar las principales exigencias asociadas a estos puestos de trabajo, la promoción de categoría, las posibilidades de intervención o de aplicación de nuevos conocimientos.

La identificación de este último tipo se concreta también por alcanzar los más bajos niveles de remuneración, el $51 \%$ ingresa menos de 60 mil ptas. al mes. Los empleadores de estos asalariados se local izan preferentemente en las empresas de más reducido tamaño, el 62\% corresponde a centros de trabajo de menos de veinticinco trabajadores, donde se alcanzan los más bajos niveles de representación laboral. La titularidad de estos centros de trabajo es privada de forma casi absoluta y destacan principal mente los subsectores de otros servicios (donde se incluye el servicio doméstico), comercio/hostelería, textil/calzado/piel, construcción y otras industrias.

Para acabar la identificación de este tipo señalaremos los rasgos característicos de las variables sociales, algunas ya avanzadas. Presenta una alta proporción de jóvenes, el $57 \%$ comprende el intervalo de dieciocho a veintinueve años y son mayoritariamente - el 54\% - mujeres. Siendo sobre todo de origen catalán, es el grupo que manifiesta una menor utilización del catalán como lengua familiar, correspondiendo por tanto en una buena proporción a la segunda generación de inmigrantes.

\subsection{H acia una tipología general de segmentación laboral para toda la población}

Con el fin de poder relacionar características del trabajo productivo con aspectos del trabajo reproductivo y de la vida cotidiana, queremos ampliar el análisis con el modelo de segmentación al conjunto de la población. Somos conscientes de que su significado es distinto, ya se trate de ocupados ya de desocupados ya de inactivos; sin embargo, dado que hay al gún grado de referencia al trabajo en casi todos los casos - porque se trabaja, se ha trabajado 0 se quiere trabajar- , hemos creído oportuno aplicar este análisis a toda la pobla ción. Sin embargo hemos mantenido un triple nivel de análisis en su construcción: el realizado de asalariados, el de los activos que realizamos a continuación y del total de la población.

Para completar el estudio de los activos es necesario validar la ubicación de dos colectivos hasta ahora no considerados: los parados y los ocupados no asalariados. En cuanto a los primeros, en nuestra base de datos no contamos, obviamente, con los elementos que nos han servido para efectuar los análisis anteriores de los asalariados y, en consecuencia, no han podido ser introducidos en el análisis. Podemos suponer que los parados, por el hecho de serlo, están en el grado máximo de la inestabilidad o bien en el segmento inferior de la segmentación. Está claro que hay diferencias dentro del colectivo de los 
parados y que muchos de ellos están en situación similar a quienes ocupan el segmento inferior o secundario de los ocupados, dado que algunos parados entran y salen de la ocupación como pasa con los de este tipo. Con todo, contemplando su situación actual, hemos preferido considerarlos a todos como un único colectivo y ubicarlos por debajo, por lo que constituyen el nuevo segmento inferior o marginal de los activos.

Este colectivo de parados abarca el $11 \%$ de las personas activas según nuestros datos; sus caracerísticas son las de los desempleados: bajo nivel de ingresos mensuales; de edad muy joven o avanzada; proporcional mente con más mujeres que hombres; de nivel de renta familiar, categoría social y nivel de estudios del entrevistado bajos. Su situación en el nivel más bajo de la segmentación justifica que sus posibilidades individuales son débiles: estudios bajos, cualificación baja, juventud o vejez y ser mujeres. También son débiles los apoyos que pueden recibir de la familia, a juzgar por la categoría económica de ésta y su renta familiar aunque ésta sea real y efectiva. Es evidente que no estamos diciendo que todos los parados tengan estas características, sino que, admitiendo que el colectivo de parados no es homogéneo - y por tanto tampoco la situación de inestabilidad y su ubicación en el segmento inferior- , sí podemos señalar que el grupo predominante es tal y como se describe. Pero se trata de un grupo que tiene un fuerte papel efectivo en el funcionamiento del mercado de trabajo. Pero es el segmento más bajo del mismo, un segmento que podríamos llamar marginal.

Respecto a los ocupados no asalariados podemos contar con datos de las variables que hemos utilizado para los asalariados. A pesar de las diferencias obvias existentes dentro de este colectivo los hemos considerado en uno sólo, puesto que desde el punto de vista de la segmentación laboral, de la estabilidad, de la capacidad de mercado, etc., podemos pensar que están todos ellos en la misma situación y sobre todo podemos muy bien suponer que todos ellos tienen mayor grado de estabilidad en el empleo que los grupos o tipos de los ocupados asalariados. Constituirá así el nuevo tipo o segmento superior dentro de la tipología de segmentación para el conjunto de los activos.

El segmento así constituido engloba al 18,1\% de los activos. Sus componentes sociales se caracterizan por la preponderancia de ingresos mensuales altos, jornada laboral el evada, edad media y alta, más hombres que mujeres, renta familiar alta, categoría del entrevistado media, diversificado según estudios, de familia totalmente catal ana o mixta. Es evidente que aquí nos encontramos ante un grupo homogéneo. Así y todo, los rasgos predominantes nos permiten hablar de un grupo sólido, a juzgar por el nivel de ingresos; con características tanto individuales (cualificación, estudios, edad, sexo) como familiares (categoría y renta familiar) que más bien acompañan a la estabilidad y a una situación de segmento superior. Por tal razón no nos parece desencaminado ubicarlos en los niveles más al tos de la segmentación, aunque este concepto sólo por analogía puede ser utilizado con respecto a ellos. D iferenciados de los estratos más altos de los asalariados, no necesariamente esto quiere decir que estén en mejor posición. 
Finalmente queremos elaborar una tipología de estratificación o segmentación laboral de toda la población mayor de dieciocho años. Es evidente que dicha tipología ha de ser también entendida en términos analógicos, puesto que grupos importantes de esa población ni trabajan ni buscan trabajo y algunos no han trabajado ni lo han buscado nunca. Esta tipología tiene sentido en esta investigación por dos razones. En primer lugar porque podemos decir que en la vida cotidiana de la mayoría de las personas el trabajo productivo tiene un papel central, bien sea porque se realiza, bien sea porque se haya trabajado y ahora no se trabaje, bien porque se desea hacerlo, bien porque se depende de él de una manera u otra y si no se trabaja es porque se hacen otras cosas (tareas reproductivas), muchas veces con finalidad productiva, o se hacen otras cosas dado que no se trabaja.

Para tener en cuenta todos los colectivos hemos de introducir dos nuevos segmentos o tipos en la clasificación, es decir, dos colectivos extraídos de las personas que están fuera del circuito laboral inmediato, se trata de dos grupos donde predominan los jubilados y las amas de casa. Por tanto, se agregarán a los seis anteriores constituyendo una tipología con ocho segmentos, estratos o tipos. Estos dos segmentos los situaremos en los niveles más bajos de la segmentación laboral, ya que ni tan siquiera están en su circuito.

El grupo de jubilados representa el 18,7\% de la población y correspondea personas que primordialmente tienen ingresos bajos, son de categoría socioeconómica baja y no tienen estudios. El grupo de amas de casa constituyen el $23,5 \%$ de la población, sus ingresos son bajos o nulos, de edad madura o joven, mujeres de renta familiar baja y en al gún grado media-baja, categoría individual baja, sin estudios o con estudios primarios, de origen geográfico diverso, residentes por todo el territorio.

D e esta forma completamos la construcción y descripción de una tipología que, centrada en el ámbito de trabajo productivo y concebida desde la perspectiva teórica de la segmentación, nos permite estructurar esta realidad social. Los resultados de la investigación que aquí hemos presentado tuvieron su continuación en fases posteriores, destinadas a profundizar en los contenidos de los tipos obtenidos y a compararlos con los resultantes en el ámbito reproductivo y del tiempo libre. En este caso se recurrió a procedimientos y métodos más intensivos orientados a captar actitudes, discursos, valores y representaciones, en definitiva, estructuras de sentido de personas originariamente ubicadas en un contexto social definido por los tipos del ámbito productivo. En otros artículos que aparecen en este número de la revista se pueden seguir las reflexiones y comentarios que apuntamos.

\section{Bibliografía}

BAgn ASCO, Arnaldo (1989). «M ercado y mercados de trabajo». Soci ología del Trabajo, 6, primavera, p. 21-31.

BenzÉCRI, J.P. (1973). L'Analyse des données. París: D unod. 
Bilbao, A.; Cachón , L.; Prieto, C. (1990). Políticas empresariales de mano de obra (Un estudio realizado en la cuenca industrializada del sur madrileño). M adrid: Comunidad de M adrid.

ButTler, F.; KEIL, H .J. (1977). «Segmentaciones del mercado de trabajo, política del mercado de trabajo y desarrollo económico regional». Revista Española de Economía, vol. 1, abril, p. 43-108.

BISQ UERRA AlzIN A, Rafael (1989). Introducción conceptual al análisis multivariable. Un enfoque informático con los paquetes SPSS-X, BM D P, LISREL y SPAD. Barcelona: Promociones y Publicaciones Universitarias.

CASSASUS, Cecilia (1980). «D el modelo neoclásico a las teorías de la segmentación del mercado de trabajo». Sociología del Trabajo, 3-4, primera época, p. 9-22.

Cornejo Álvarez, José M anuel (1988). Técnicas de investigación social: el análisisde correspondencias (Teoría y Práctica). Barcelona: Promociones y Publicaciones Universitarias.

CH AN D ON, J.L.; PIn SO N, S. (1981). Analyse typologique. Théories et applications. París: M asson.

D O ERIN GER, P.B.; PIORE, M .J. (1983a). «Los mercados internos detrabajo». En TOHARIA, L. (ed.). El mercado de trabajo: teoría y aplicaciones. M adrid: Alianza, p. 341-388.

- (1983b). «El paro y el mercado dual de trabajo». En T O H ARIA, L. (ed.). El mercado de trabajo: teoría y aplicaciones. M adrid: Alianza, p. 307-320.

- (1985). M ercados internos de trabajo y análisislaboral. M adrid: M inisterio de Trabajo y Seguridad Social.

EdWARDS, R.C.; G ORD ON, D .M .; REICH, M . (eds.) (1975). Labor M arket Segmentation. Lexington (M assachusets): Lexington Books.

Escofier, B.; PAGES, J. (1988). Analyses factorielles simples et multiples. París: D unod.

FERn ÁN D EZ SANTANA, O . (1991). «El análisis de cluster: aplicación, interpretación y validación». Papers. Revista de Sociologia, 37, p. 65-76.

GoRD ON, D .M .; EdWARDS, R.C.; REICH, M . (1986). Trabajo segmentado, trabajadores divididos. La transformación histórica del trabajo en los Estados U nidos. M adrid: $M$ inisterio de Trabajo y Seguridad Social.

Grup d'Estudis So ciològics SO bre La Vida Q uotidiana I el Treball (1994). Transformaciones del trabajo y bienestar social. Informe del proyecto de investigación financiado por la DGICYT.

KERN, H .; SCHUM AN N, M . (1988). El fin dela división del trabajo. Racionalización en la producción industrial. M adrid: M inisterio de Trabajo y Seguridad Social.

LEBART, Ludovic (1977). «La validité des résultats en analyse des données». Consommation, 1, enero-marzo, p. 41-69.

Lebart, L.; M ORIN EAU, A.; Fen ELO N, J.P. (1985). Tratamiento estadístico de datos. M étodos y programas. Barcelona: $M$ arcombo.

Lebart, L.; M ORIN EAU, A.; LAM BeRT, T. (1987). SPAD .N : Système Portable pour I'Analyse des $D$ onnées. Version 1.0. M anuel de Référence. Sèvres: CISIA.

LeFEBVRE, Jacques (1983). Introduction aux analyses statistiques multidimensionnelles París: M asson.

LOPE PEÑ A, Andreu (1996). Innovación tecnológica y cualificación: la polarización de las cualificaciones en la empresa. M adrid: Consejo Económico y Social.

Ló PEZ ROLDÁN, Pedro (1994). La construcción de tipologías en Sociología: propuesta metodológica de construcción, análisis y validación. A plicación al estudio de la segmentación del mercado de trabajo en la Regió M etropolitana de Barcelona. Tesis doctoral. Bellaterra: U niversitat Autònoma de Barcelona. 
- (1996a). «La construcción de tipologías: metodología de análisis». Papers. Revista de Sociologia, 48, p. 9-29.

- (1996b). «La construcción de una tipología de segmentación del mercado de trabajo». Papers. Revista de Sociologia, 48, p. 41-58.

LozARes Colin A, C.; Ló Pez RoldÁn, P. (1991a). «El análisis multivariado: definición, criterios y clasificación». Papers. Revista de Sociologia, 37, p. 9-29.

- (1991b). «El análisis de componentes principales: aplicación al análisis de datos secundarios». Papers. Revista de Sociologia, 37, p. 31-63.

- (1991c). «El muestreo estratificado por análisis multivariado». En: LATIESA, M. (ed.). El pluralismo metodológico en la investigación social: ensayos típicos. Granada: U niversidad de Granada, p. 107-160.

M ART Ín ARTILES, Antonio (1992). «Proceso de flexibilización y modelo de relaciones laborales postfordista. Estudio comparado de casos». Sociología del Trabajo, 15, primavera, p. 63-90.

M aruani, M.; ReYnaud, E. (comp.) (1991). D ebates sobreed empleo. Francia y Alemania. $M$ adrid M inisterio de Trabajo y Seguridad Social.

M IGUÉLEZ Lo Bo, Faustino (1989). La segmentación del mercado de trabajo. M imeo.

M IGUÉLEZ LoBO, F.; Ló PEZ RoldÁn, P. (1988). Enqueta M etropolitana 1986. Condicions de vida i hàbits de la població de l'àrea metropolitana de Barcelona. La pobresa a l'àrea metropolitana de Barcelona. Barcel ona: Institut d'Estudis M etropolitans de Barcelona.

- (1989). «El trabajo en la pequeña empresa española. ¿U na perspectiva alternativa?». Papers. Revista de Sociologia, 32, p. 63-87.

M Iguélez Lo bo, F.; Prieto Rodríguez, C. (1991). Las relacioneslaborales en España. $M$ adrid: Siglo XXI.

M IGUÉlez LoBo, F.; T ORns M ARTín, T. (1992). Enquesta de la Regió M etropolitana de Barcel ona 1990. Treball, condicions econòmiquesi formes de consum. Barcelona: M ancomunitat de M unicipis del'Àrea M etropolitana de Barcelona i Diputació de Barcelona.

O STERM AN, Paul (comp.) (1988). Los mercadosinternos de trabajo. M adrid: M inisterio de Trabajo y Seguridad Social.

PAHL, R.E. (1990). «D el'"economia informal" a "formes de treball": models i tendències transnacionals». Papers. Revista de Sociologia, 34, p. 63-95.

- (1991). Divisiones del trabajo. M adrid: M inisterio de Trabajo y Seguridad Social.

PIORE, M ichael J. (1983a). Paro einflación. M adrid: Alianza.

- (1983b). «La importancia de la teoría del capital humano para la economía del trabajo; un punto de vista disidente». En TOH ARIA, L. (ed.). El mercado de trabajo: teoría y aplicaciones. M adrid: Alianza, p. 105-114.

- (1983c). «Los fundamentos tecnológicos del dualismo y de la discontinuidad». En T o H ARIA, L. (ed.). El mercado de trabajo: teoría y aplicaciones. M adrid: Alianza, p. 255-282.

- (1983d). «El dualismo como respuesta al cambio y la incertidumbre». En T O HARIA, L. (ed.). El mercado de trabajo: teoría y aplicaciones. M adrid: Alianza, p. 223-254.

- (1983e). «N otas para una teoría de la estratificación del mercado de trabajo». En To o ARIA, L. (ed.). El mercado de trabajo: teoría y aplicaciones. M adrid: Alianza, p. 193-222.

PIORE, M .J.; SABEL, Ch. (1990). La segunda ruptura industrial. M adrid: Alianza.

Prieto Rodríguez, Carlos (1989). «iM ercado de trabajo?». Revista Española de Investigaciones Sociológicas, 47, p. 177-192. 
RECIO, Albert (1988). Capitalismo y formas de contratación laboral. M adrid: M inisterio de Trabajo y Seguridad Social.

- (1991). «La segmentación del mercado de trabajo en España». En M IGUÉLEZ, F.; PRIETO, C. (eds.). Las relaciones laborales en España. M adrid: Siglo XXI, p. 97-116.

SÁN CH EZ C ARRIón, Juan Javier (1984). Introducción a las técnicas de análisis multivariable aplicadas a las ciencias sociales. M adrid: Centro de Investigaciones Sociológicas.

SANTILlana del Barrio, I.; M ayCas Tarascón, J. (1979). «La división del mercado de trabajo: el problema de los desaventajados». Cuadernos de Ciencias Económicas y Empresariales, 5, p. 17-43.

SEN GEN BERGER, Werner (comp.) (1988). Lecturas sobre el mercado de trabajo en la República Federal de Alemania. M adrid: M inisterio de Trabajo y Seguridad Social.

To H ARIA, Luis (comp.) (1983). El mercado de trabajo: Teorías y aplicaciones. M adrid: Alianza.

T O Rn S M ART ín, Teresa (1991). «Trabajo, mujer y clase obrera». Trabajo y dase obrera hoy. Fundación de Investigaciones $M$ arxistas.

ToRn S M ART ín, T.; CARRASQ UER O TO, P. (1987). «En torn dels conceptes de dona i treball a C atalunya». Visió de Catalunya. Barcelona: D iputació de Barcelona, p. 235-247.

VILLA, Paola (1990). La estructuración de los mercados de trabajo. La siderurgia y la cons trucción en Italia. M adrid: M inisterio de Trabajo y Seguridad Social.

Vo LLE, M ichel (1985). Analyse des données. París: Economica.

W ILKIN SO N, Frank (ed.) (1981). The D ynamics of Labour M arket Segmentation. Londres: Academic Press. 